\title{
Neutron Star Mergers, Disks Around Black Holes, and Gamma-Ray Bursts
}

\author{
H.-Th. Janka and M. Ruffert \\ Max-Planck-Institut für Astrophysik, D-85740 Garching, Germany
}

\begin{abstract}
We have performed three-dimensional hydrodynamical simulations of the coalescence of binary neutron stars taking into account the emission and backreaction of gravitational waves in the Newtonian code based on the "Piecewise Parabolic Method". The use of the physical equation of state (EOS) of Lattimer \& Swesty (1991) allowed us to calculate the production of neutrinos. We evaluated our models for the efficiency of $\nu \bar{\nu}$ annihilation in the surroundings of the coalescing neutron stars. The corresponding energy deposition prior to and during merging turned out to be $2-3$ orders of magnitude too small to power a typical $\gamma$-ray burst (GRB) with an energy output of $\sim\left(10^{51} / 4 \pi\right)$ erg/sterad at cosmological distances. Analytical estimates of the subsequent evolution of the disk which possibly surrounds the central black hole showed that even under the most favorable conditions the energy provided by $\nu \bar{\nu} \rightarrow e^{-} e^{+} \rightarrow \gamma \gamma$ falls short by at least an order of magnitude. We discuss the implications of our results and speculate about possibilities how $\nu \bar{\nu}$ annihilation might still be a viable energy source for GRBs.
\end{abstract}

\section{Introduction}

The distribution of 1122 observed GRBs as reported by the BATSE 3B Catalog is isotropic over the sky and non-uniform in distance (Meegan et al. 1995). One of the currently most popular models for the origin of the $\gamma$ emission is the merging of two neutron stars (NS-NS) or of a neutron star and a black hole (NS-BH) at cosmological distances (Paczyński 1986, Goodman 1986, Eichler et al. 1989, Narayan et al. 1991). These should occur at a rate of $10^{-4}-10^{-5} \mathrm{yr}^{-1}$ per galaxy and thus with a sufficiently large frequency to explain the observed burst rate (Phinney 1991, Narayan et al. 1991). Only about $1 \%$ of the several $10^{53} \mathrm{erg}$ of gravitational binding energy released in the merging would be enough to power a burst, and the compactness of the source might account for the variations in the $\gamma$-ray flux on timescales as short as $1 \mathrm{~ms}$.

The non-thermal $\gamma$-rays are supposed to be produced by a fireball of relativistic $e^{-} e^{+}$pairs that are created by the annihilation of $\nu \bar{\nu}$ pairs in the vicinity of the hot, merged object (Goodman et al. 1987). However, the large $\nu$ fluxes also drive a baryonic wind by energy deposition near the neutrinosphere (Duncan et al. 1986, Woosley \& Baron 1992) which "pollutes" the cloud of $\gamma$ 's and $e^{-} e^{+}$pairs. The baryonic load prevents relativistic expansion and keeps the flow optically thick, leading to a degradation of the $\gamma$-rays. Thus the energy release 
will be mainly kinetic rather than in photons (Paczyński 1990). If spherical symmetry is broken, as in the case of the merger scenario where a rapidly rotating disk can be formed and material is pulled away from the rotation axis by centrifugal forces, then a baryon-free funnel along the rotation axis may allow relativistic beams of $\gamma$ 's and $e^{-} e^{+}$to escape (Mészáros \& Rees 1992, Mochkovitch et al. 1993, Woosley 1993). Annihilation of $\nu \bar{\nu}$ along the system axis might lead to matter ejection with Lorentz factors of $\Gamma \sim 10^{2}-10^{3}$ (Mochkovitch et al. 1995) which are in the right range to enable copious $\gamma$ production during shock interaction with ambient interstellar gas (Rees \& Mészáros 1992).

\section{Numerical approach}

The $\nu$ emission and $\nu \bar{\nu}$ annihilation during the coalescence of binary NSs were investigated. To this end the $3 \mathrm{D}$ Newtonian equations of hydrodynamics were integrated by the Riemann-solver based "Piecewise Parabolic Method" (Colella \& Woodward 1984) on an equidistant Cartesian grid with a resolution of $64 \times 64 \times 32$ or $128 \times 128 \times 64$ zones. The effects of the backreaction of gravitational waves on the hydrodynamic flow were included (see Ruffert et al. 1996a). The properties of neutron star matter were described by the EOS of Lattimer \& Swesty (1991). Energy loss and changes of the electron abundance due to the emission of $\nu$ 's were taken into account by an elaborate "neutrino leakage scheme" that was calibrated using diffusion results (Ruffert et al. 1996a). Neutrinos of all types are produced by thermal processes and $\nu_{e}$ and $\bar{\nu}_{e}$ also by the $\beta$-processes. Neutrino opacities and diffusion timescales were calculated from $\nu-n, p$ scattering and the inverse $\beta$-reactions (Ruffert et al. 1996a).

We have simulated the coalescence of two identical, cool (initial central temperature $k_{\mathrm{B}} T_{\mathrm{c}} \approx 7 \mathrm{MeV}$ ) neutron stars with a baryonic mass of about $1.6 M_{\odot}$, a radius of $15 \mathrm{~km}$, and an initial center-to-center distance of $42 \mathrm{~km}$ for three different cases of initial neutron star spins. Initial orbital and spin periods were $\sim 2.6 \mathrm{~ms}$. We evaluated our models for the efficiency of $\nu \bar{\nu}$ annihilation and investigated the question whether the corresponding energy deposition is powerful enough to account for the energetics of burst events at cosmological distances. That is the crucial question when associating GRBs with NS-NS or NS-BH mergers, independent of the detailed, very complex processes which lead to the emission of observed $\gamma$-rays.

\section{Results}

The total $\nu$ luminosity prior to and during the dynamical phase of the coalescence is very small $\left(L_{\nu} \lesssim 10^{51} \mathrm{erg} / \mathrm{s}\right)$, becomes about $1-2 \cdot 10^{52} \mathrm{erg} / \mathrm{s}$ when the stars have merged into one rapidly spinning massive body, and climbs to $1-1.5 \cdot 10^{53} \mathrm{erg} / \mathrm{s}$ after spun off material has formed a hot toroidal cloud with a mass of $0.1-0.2 M_{\odot}$ around the wobbling and pulsating central object. The $\nu$ fluxes are clearly dominated $(\sim 90-95 \%)$ by the emission from this "disk". Since the hot disk matter is $n$-rich, $\bar{\nu}_{e}$ are radiated with a luminosity that is a factor 3-6 higher than the (individual) luminosities of $\nu_{e}$ and $\nu_{x}\left(\equiv \nu_{\mu}, \bar{\nu}_{\mu}, \nu_{\tau}, \bar{\nu}_{\tau}\right)$. The mean energies of the emitted $\nu$ 's are very similar to those of supernova neutrinos, $\left\langle\epsilon_{\nu_{e}}\right\rangle \approx 12 \mathrm{MeV},\left\langle\epsilon_{\bar{\nu}_{e}}\right\rangle \approx 20 \mathrm{MeV}$, and $\left\langle\epsilon_{\nu_{x}}\right\rangle \approx 27 \mathrm{MeV}$. 
Table 1. Summary of the phases of $\nu$ emission during NS-NS merging. $t_{\mathrm{ph}}$ is the duration of the phase, $T$ the maximum temperature in the stars or the merged object, $L_{\nu}^{\text {tot }}$ the total $\nu$ luminosity (in all kinds of $\nu$ 's), $\left\langle\epsilon_{\nu}\right\rangle$ the average energy of the emitted $\nu$ 's (mean value for all species), $E_{\nu}$ the total energy radiated in $\nu$ 's during the different phases, $\dot{E}_{\nu \bar{\nu}}$ the integral energy deposition rate by $\nu \bar{\nu}$ annihilation in the surroundings of the merging stars, and $E_{\nu \bar{\nu}}$ the integral energy deposition by $\nu \bar{\nu}$ annihilation. The data for the pre-merging phase (defined as the phase where the binary NSs are so close that significant tidal deformation and dissipation can occur), the dynamical merging phase, and the post-merging phase were obtained from the numerical models, whereas the data of the torus or disk phase were estimated from a simple analytical model for a $\sim 3 M_{\odot} \mathrm{BH}$ with an $\sim 0.1 M_{\odot}$ accretion torus (Janka \& Ruffert 1996, Ruffert et al. 1996b).

\begin{tabular}{lccccccc}
\hline Phase & $\begin{array}{c}t_{\mathrm{ph}} \\
(\mathrm{ms})\end{array}$ & $\begin{array}{c}k_{\mathrm{B}} T \\
(\mathrm{MeV})\end{array}$ & $\begin{array}{c}L_{\nu}^{\text {tot }} \\
(\mathrm{erg} / \mathrm{s})\end{array}$ & $\begin{array}{c}\left\langle\epsilon_{\nu}\right\rangle \\
(\mathrm{MeV})\end{array}$ & $\begin{array}{c}E_{\nu} \\
(\mathrm{erg})\end{array}$ & $\begin{array}{c}\dot{E}_{\nu \tilde{\nu}} \\
(\mathrm{erg} / \mathrm{s})\end{array}$ & $\begin{array}{c}E_{\nu \bar{\nu}} \\
(\mathrm{erg})\end{array}$ \\
\hline pre-merging & few & $\lesssim 1$ & $\lesssim 10^{48}$ & $\lesssim 1$ & $\lesssim 10^{46}$ & - & - \\
merging & $\sim 1$ & $\sim 10$ & $<10^{52}$ & $\sim 20$ & $\lesssim 10^{49}$ & $\sim 10^{48}$ & $\sim 10^{45}$ \\
post-merging & $\lesssim 10$ & $\lesssim 60$ & $\sim 10^{53}$ & $\sim 20$ & $\sim 10^{51}$ & $\lesssim 4 \cdot 10^{50}$ & $\lesssim 4 \cdot 10^{48}$ \\
BH with disk & $\sim 100$ & $\sim 30$ & $\sim 10^{53}$ & $\sim 20$ & $\sim 10^{52}$ & $<10^{51}$ & $<10^{50}$ \\
\hline
\end{tabular}

When the $\nu$ luminosities are highest, only about $0.2-0.3 \%$ of the energy emitted in $\nu$ 's is deposited in the immediate neighborhood of the merger by $\nu \bar{\nu}$ annihilation, and the maximum integral energy deposition rate is found to be $\sim$ $3-4 \cdot 10^{50} \mathrm{erg} / \mathrm{s}$. Thus, to pump an energy of the order of $10^{51} /(4 \pi) \mathrm{erg} / \mathrm{steradian}$ into a fireball of $e^{+} e^{-}$-pairs and photons, the strong $\nu$ emission would have to continue for several seconds. Since a collapse of the central core of the merger with a mass of $\gtrsim 3 M_{\odot}$ into a $\mathrm{BH}$ within milliseconds seems unavoidable, we conclude that the available energy is insufficient by a factor of about 1000 to explain GRBs at cosmological distances. However, it appears possible that an accretion torus with a mass of $\sim 0.1-0.2 M_{\odot}$ remains around the central $\mathrm{BH}$ and is accreted on the timescale of viscous angular momentum transport which is $\sim 0.1 \mathrm{~s}$ (Table 1). Analytical estimates suggest that even under the most favorable conditions in this torus and with an optimum value of the disk viscosity, annihilation of emitted $\nu \bar{\nu}$ pairs provides an energy that is still more than a factor of 10 too small to account for powerful cosmological GRBs, unless focussing of the fireball expansion plays an important role (Table 1; details in: Janka \& Ruffert 1996, Ruffert et al. 1996b; see also Jaroszyński 1993, 1996).

\section{Discussion and conclusions}

To rescue $\nu \bar{\nu}$ annihilation as a possible energy source for GRBs from NS-NS or NS-BH mergers, more extreme physics than used in our models has to be considered. Although not suggested by current models for the state of matter 
above nuclear matter density, one might speculate about a stiffer EOS at very high densities. Together with the support by centrifugal forces and thermal pressure, the maximum mass of stable rotating configurations might be large enough to prevent the merged object from gravitational collapse for a time of several seconds, in particular if one considers the merging of two neutron stars with baryon masses of only 1.3-1.4 $M_{\odot}$ (gravitational masses 1.1-1.3 $M_{\odot}$ ). The upper limit for $E_{\nu \bar{\nu}}$ in Table 1 (which is in fact extreme because it was derived by making a whole chain of favorable assumptions, see Ruffert et al. 1996b) is so close to the desired value to explain GRBs that one is tempted to consider scenarios or situations where slightly larger torus masses and/or larger BH masses (both increase $E_{\nu \bar{\nu}}$, see Janka \& Ruffert 1996) emerge. Could mergings of NSs with BHs of masses of a few $M_{\odot}$ produce such systems? What happens when a rotating massive star undergoes stellar core collapse but fails to produce a supernova (Woosley 1993)? Does a Kerr BH form at the center, a case in which the disk would be accreted onto the $\mathrm{BH}$ on a much longer timescale and much more energy could be radiated in $\nu$ 's than given in Table 1 and Janka \& Ruffert (1996) (see Jaroszyński 1996)? Is there a possibility to beam the $e^{+} e^{-} \gamma$ fireball significantly? Which role do magnetic fields play in this context? Are the $\nu$ luminosities from the $\nu$-opaque accretion torus enhanced by convective overturn processes? What happens in NS collisions?

Acknowledgments. The Deutsche Forschungsgemeinschaft (SFB 375 Particle-Astrophysics) has partially supported the participation in the IAU Coll. 163.

\section{References}

Colella P., Woodward P.R., 1984, JCP 54, 174

Duncan R.C., Shapiro S.L., Wasserman I., 1986, ApJ 309, 141

Eichler D., Livio M., Piran T., Schramm D.N., 1989, Nat 340, 126

Goodman J., 1986, ApJ 308, L47

Goodman J., Dar A., Nussinov S., 1987, ApJ 314, L7

Janka H.-Th., Ruffert M., A\&A 307, L33

Jaroszyński M., 1993, Acta Astron. 43, 183

Jaroszyński M., 1996, A\&A 305, 839

Lattimer J.M., Swesty F.D., 1991, Nucl. Phys. A535, 331

Meegan C.A., et al., 1995, ApJS, in press

Mészáros P., Rees M.J., 1992, MNRAS 257, 29P

Mochkovitch R., Hernanz M., Isern J., Martin X., 1993, Nat 361, 236

Mochkovitch R., Hernanz M., Isern J., Loiseau S., 1995, A\&A 293, 803

Narayan R., Piran T., Shemi A., 1991, ApJ 379, L17

Paczyński B., 1986, ApJ 308, L43

Paczyński B., 1990, ApJ 318, 363

Phinney E.S., 1991, ApJ 380, L17

Rees M.J., Mészáros P., 1992, MNRAS 258, 41P

Ruffert M., Janka H.-Th., Schäfer G., 1996a, A\&A 311, 532

Ruffert M., Janka H.-Th., Takahashi K., Schäfer G., 1996b, A\&A, in press

Woosley S.E., Baron E., 1992, ApJ 391, 228

Woosley S.E., 1993, ApJ 405, 273 